\title{
Avaliação dos níveis de zeólita em dietas para suínos em fase de crescimento e terminação ${ }^{1}$
}

\author{
Gabriel Cipriano Rocha², Juarez Lopes Donzele², Rita Flávia Miranda de Oliveira², Francisco \\ Carlos de Oliveira Silva ${ }^{3}$, Charles Kiefer ${ }^{4}$, Paulo César Brustolini ${ }^{2}$, Cinthia Maria Carlos \\ Pereira $^{2}$, Leandro Alebrante ${ }^{2}$
}

\author{
${ }^{1}$ Research supported by Indústria Celta Brasil Ltda. \\ 2 DZO/UFV. \\ ${ }^{3}$ EPAMIG. \\ ${ }^{4}$ DZO/UFMS
}

RESUMO - Este estudo foi realizado com o objetivo de avaliar níveis de zeólita em dietas para suínos e seus efeitos no desempenho, nas características de carcaça e nos parâmetros sanguíneos e histopatológicos do parênquima hepático desses animais nas fases de crescimento e terminação. Foram utilizados 84 suínos machos castrados, híbridos comerciais de alto potencial genético para deposição de carne, com peso inicial de $30,34 \pm 1,14 \mathrm{~kg}$, distribuídos em delineamento experimental de blocos ao acaso, com sete níveis de adsorvente $(0,0-0,0 ; 1,0-0,5 ; 1,0-1,0 ; 2,0-1,0 ; 2,0-2,0 ; 3,0-1,5 ; 3,0-3,0$, respectivamente, nas fases de crescimento e terminação), com seis repetições e dois animais por baia (unidade experimental). Utilizou-se o sorgo como fonte de contaminação natural por micotoxinas. Os níveis de adsorvente das dietas experimentais foram obtidos a partir da inclusão de zeólita em substituição à areia lavada das dietas sem adsorvente. Não houve efeito dos níveis de zeólita nas dietas sobre o consumo diário de ração, o ganho de peso diário, a conversão alimentar e as características carcaça avaliadas. Os níveis de zeólita nas dietas também não influenciaram os parâmetros sanguíneos e histopatológicos nem os teores ósseos de cálcio e fósforo dos suínos. Devido ao bom índice de desempenho e às características de carcaça, bem como à normalidade dos outros parâmetros estudados dos animais que receberam dieta sem adsorvente, pode-se inferir que as dietas continham baixo grau de contaminação por micotoxinas. Os níveis de zeólita avaliados não influenciam o desempenho, as características de carcaça nem os parâmetros sanguíneos e histopatológicos do parênquima hepático de suínos nas fases de crescimento e terminação recebendo dietas com baixo nível de contaminação por micotoxinas.

Palavras-chave: adsorvente, fungos, micotoxinas, nutrição

\section{Evaluation of zeolite levels in diets for swine in the growing and finishing phases}

\footnotetext{
ABSTRACT - This study was conducted to evaluate levels of inclusion of zeolite in diets for pigs on performance, carcass characteristics, blood and liver histopathological parameters of these animals in their growing-finishing phases. Eightyfour barrows from commercial hybrids of high genetic potential for meat deposition, with initial weight of $30.34 \pm 1.14 \mathrm{~kg}$ were distributed in randomized blocks design, with seven levels of adsorbent $(0.0-0.0 ; 1.0-0.5 ; 1.0-1.0 ; 2.0-1.0 ; 2.0-2.0$; 3.0-1.5; 3.0-3.0, respectively, on growing and finishing phases), six replicates with two animals per pen, which was considered the experimental unit. Sorghum was used as naturally contaminated source of mycotoxins. The different levels of adsorbent in the diets were obtained by the supplementation of zeolite in the basal diet. The inclusion of zeolite in the diet did not affect the daily feed intake, daily weight gain, feed conversion or the carcass characteristics of the pigs. In the same way blood parameters, liver histopathological examination and the bone concentration neither calcium nor phosphorus were affected by the different levels of zeolita. Due to the good performance and carcass characteristics, as well as the normality of the other parameters of the animals fed diets without adsorbents analyzed, it can be inferred that the diets had low levels of mycotoxin contamination. The levels of zeolite do not affect the performance, carcass characteristics, blood parameters or liver histopathological examination in growing-finishing pigs fed diets with low level of contamination by mycotoxin.
}

Key Words: adsorbent, fungi, mycotoxins, nutrition

Recebido em 13/7/2010 e aprovado em 13/4/2011.

Correspondências devem ser enviadas para: gcr90@yahoo.com.br 


\section{Introdução}

No contexto da estabilidade e rentabilidade da produção de suínos, o item alimentação, por ser o fator que mais onera o custo de produção dos animais, tem merecido atenção especial. Assim, qualquer fator que possa comprometer a eficiência de utilização do alimento pelo animal pode refletir negativamente no sucesso do empreendimento. Entre os problemas inerentes à alimentação, destaca-se a presença de micotoxinas. A contaminação das dietas por micotoxinas tem sido considerada responsável pela redução do desempenho dos animais em sistema de produção onde os demais fatores, como manejo e instalações, estejam adequados (Jones, 2005).

O termo micotoxina tem sido utilizado para definir um grupo de metabólitos secundários produzidos por fungos e que induzem uma série de reações tóxicas no organismo. Esses fungos podem se desenvolver nos alimentos no campo ou durante o armazenamento. Vários autores têm associado o baixo desempenho de suínos quando alimentados com dietas contaminadas por micotoxinas (Muller et al., 1999; Agag, 2004a; Rustemeyer et al., 2010).

A ingestão de dietas contaminadas, além de ocasionar redução no crescimento e no desenvolvimento, torna os animais mais susceptíveis às enfermidades (Dersjant-Li et al., 2003). Além disso, as micotoxinas podem prejudicar o valor nutricional dos alimentos, principalmente quanto ao nível de energia (Büzen \& Haese, 2006). Em baixas concentrações, as micotoxinas não têm efeito aparente sobre o rebanho (Accensi et al., 2006), mas podem gerar resíduos nos produtos de origem animal (Murphy et al., 2006), evidenciando a importância da inclusão de adsorventes nas dietas.

Por sua vez, os adsorventes têm sido utilizados em dietas para suínos devido às suas propriedades de atuar como sequestrantes de micotoxinas, o que pode influenciar de forma positiva a saúde e o desempenho dos animais. Entre os adsorventes, estão as zeólitas, que são aluminossilicatos hidratados caracterizados pela alta superfície interna e alta capacidade de troca catiônica e que possuem a habilidade de se aderir à toxina e impedir sua absorção pelo trato gastrointestinal tornando-a inerte e não tóxica para os animais (Lindemann et al., 1993). A pesquisa foi realizada com o objetivo de avaliar níveis de zeólita em dietas para suínos e seus efeitos sobre o desempenho, as características de carcaça e os parâmetros sanguíneos e histológicos do parênquima hepático desses animais nas fases de crescimento e terminação.

\section{Material e Métodos}

O experimento foi realizado no Galpão Experimental do Setor de Suinocultura da Fazenda Experimental Vale do Piranga da EPAMIG, em Oratórios, Minas Gerais, no período de fevereiro a abril de 2009.

Foram utilizados 84 suínos machos castrados, híbridos comerciais de alto potencial genético para deposição de carne, com 70 dias de idade e peso inicial de $30,34 \pm 1,14 \mathrm{~kg}$, distribuídos em delineamento experimental de blocos ao acaso, com sete níveis de adsorvente ( $0,0-0,0 ; 1,0-0,5 ; 1,0-1,0$; $2,0-1,0 ; 2,0-2,0 ; 3,0-1,5 ; 3,0-3,0$, respectivamente, nas fases de crescimento e terminação), com seis repetições e dois animais por baia, que foi considerada unidade experimental. Na formação dos blocos, levou-se em consideração o peso inicial dos animais.

As dietas experimentais para as fases de crescimento (70 aos 105 dias) e terminação (106 aos 135 dias) foram formuladas à base de milho, sorgo baixo tanino e farelo de soja e suplementadas com minerais, vitaminas e aminoácidos (Tabela 1) para atender às exigências nutricionais dos animais, descritas por Rostagno et al. (2005). As relações aminoacídicas entre a lisina e os demais aminoácidos essenciais foram atendidas de acordo com o padrão de proteína ideal estabelecido por Rostagno et al. (2005). Utilizou-se o sorgo como fonte de contaminação natural por micotoxinas. Os níveis de adsorvente das dietas experimentais foram obtidos por meio da inclusão de zeólita em substituição à areia lavada das dietas sem adsorvente.

As rações e a água foram fornecidas à vontade aos animais durante o período experimental. Os resíduos de ração do chão foram coletados diariamente, pesados semanalmente e somados às sobras do comedouro no final do período experimental.

Os animais foram alojados em galpão de alvenaria com piso de concreto, coberto com telhas de amianto. As baias continham comedouros semiautomáticos, bebedouros tipo chupeta e dispunham de área de $1,87 \mathrm{~m}^{2} /$ animal. Durante o período experimental, foram registradas diariamente as temperaturas de máxima e mínima, por meio de um termômetro instalado no centro do galpão.

Foram realizadas pesagens dos animais no início e ao final do período experimental para determinações do consumo diário de ração, do ganho diário de peso e da conversão alimentar. Paralelamente, foram coletadas amostras de sangue no início e ao final do período experimental, mediante venipunção no sinus orbital, utilizando-se agulhas hipodérmicas $40 \times 16$ e frascos vacutainer. Após a coleta de sangue, os frascos foram 
Tabela 1 - Composição das dietas experimentais

\begin{tabular}{|c|c|c|}
\hline Ingrediente & Crescimento & Terminação \\
\hline Milho & 30,351 & 35,079 \\
\hline Farelo de soja & 30,760 & 27,192 \\
\hline Sorgo baixo tanino & 30,000 & 30,000 \\
\hline Óleo de soja & 2,993 & 2,497 \\
\hline Areia lavada & 3,185 & 3,100 \\
\hline Fosfato bicálcico & 1,218 & 0,778 \\
\hline Calcário & 0,624 & 0,543 \\
\hline Butil-hidroxi-tolueno & 0,010 & 0,010 \\
\hline L-lisina $\mathrm{HCl}$ & 0,150 & 0,100 \\
\hline DL-metionina & 0,066 & 0,042 \\
\hline L-treonina & 0,020 & 0,003 \\
\hline Suplemento vitamínico ${ }^{1}$ & 0,100 & 0,100 \\
\hline Suplemento mineral ${ }^{2}$ & 0,050 & 0,050 \\
\hline Sal comum & 0,408 & 0,356 \\
\hline Promotor de crescimento & 0,065 & 0,150 \\
\hline Zeólita ${ }^{3}$ & 0,000 & 0,000 \\
\hline \multicolumn{3}{|l|}{ Composição nutricional calculada ${ }^{4}$} \\
\hline Energia metabolizável (kcal/kg) & 3.230 & 3.230 \\
\hline Proteína bruta (\%) & 19,41 & 18,11 \\
\hline Lisina digestível (\%) & 1,000 & 0,880 \\
\hline Treonina digestível (\%) & 0,650 & 0,590 \\
\hline Metionina + cistina digestível (\%) & 0,600 & 0,550 \\
\hline Triptofano digestível (\%) & 0,211 & 0,190 \\
\hline Valina digestível (\%) & 0,817 & 0,760 \\
\hline Sódio (\%) & 0,180 & 0,160 \\
\hline Cálcio (\%) & 0,630 & 0,484 \\
\hline Fósforo disponível (\%) & 0,332 & 0,248 \\
\hline \multicolumn{3}{|c|}{ 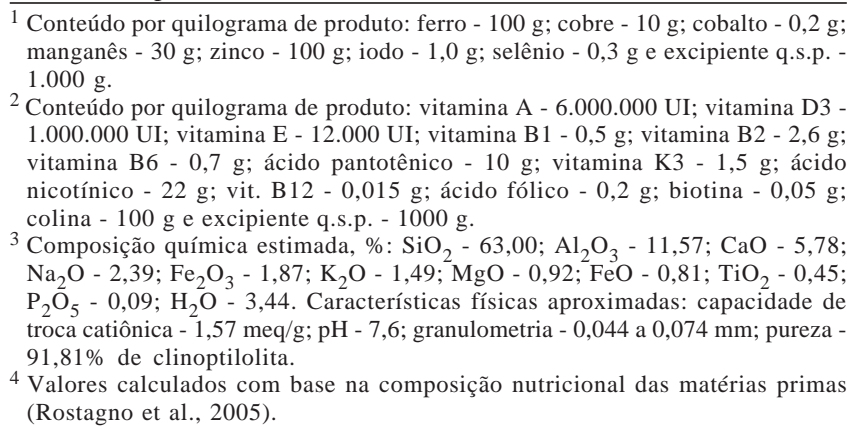 } \\
\hline
\end{tabular}

encaminhados imediatamente ao laboratório clínico. Para obtenção de soro sanguíneo, foram colhidos $10 \mathrm{~mL}$ de sangue de cada animal, em frascos a vácuo sem anticoagulante.

Após a coleta, as amostras foram mantidas em repouso à temperatura ambiente $\left(16,6\right.$ a $\left.29,6{ }^{\circ} \mathrm{C}\right)$ até a retração do coágulo e posterior centrifugação a $700 \mathrm{G}$ (10.000 rpm) por 10 minutos, sendo o soro sanguíneo retirado por pipetagem para a dosagem de gamaglutamiltransferase, aspartato aminotransferase, alanina aminotransferase, fosfatase alcalina, proteína total, albumina, creatinina e ureia. O plasma foi obtido de amostras de $5 \mathrm{~mL}$ de sangue colhidas em frascos siliconizados contendo fluoreto de sódio, as quais foram centrifugadas em velocidade e tempo semelhantes às utilizadas para o soro, imediatamente após a coleta, para mensuração da glicose. As mensurações sanguíneas foram realizadas por meio de equipamento eletrônico utilizando-se reagentes comerciais.
Ao final do período experimental, todos os animais foram mantidos em jejum alimentar por aproximadamente 15 horas, foram pesados e embarcados em caminhão e transportados para o frigorífico Vale do Piranga, localizado no município de Ponte Nova, Minas Gerais. No frigorífico, os suínos foram alojados em baias coletivas de espera com acesso à vontade a água. Por ocasião do abate, os animais foram insensibilizados por eletronarcose e, posteriormente, sangrados, escaldados e eviscerados.

Na linha de abate, as carcaças foram avaliadas individualmente com auxílio de pistola tipificadora StorkSFK, utilizando-se o sistema informatizado "Fat-o-Meater Fom”. A pistola foi introduzida na altura da $3^{\text {a }}$ vértebra dorsal, transpassando o toucinho e o músculo longissimus dorsi, conforme metodologia adotada pelo frigorífico. Foram obtidos os dados de peso da carcaça quente, espessura de toucinho, profundidade de lombo, porcentagem e quantidade de carne magra na carcaça. Além disso, foi determinado o rendimento de carcaça pela relação entre o peso da carcaça quente e o peso dos animais em jejum.

As análises histolopatológicas do parênquima hepático foram realizadas no setor de Histopatologia do Hospital Veterinário da Universidade Federal de Viçosa (UFV). Foram colhidos fragmentos dos diversos lobos hepáticos de $1 \mathrm{~cm}$ de diâmetro, que foram fixados em formol 10\% neutro tamponado $\mathrm{pH}$ 7,2 por 24 horas; em seguida, as amostras foram desidratadas em soluções alcoólicas crescentes 70 , 80, 90, 100\% I e II e, logo após, foram diafanizadas em xilol e incluídas em parafina. As amostras foram, então, cortadas em micrótomo calibrado para cortes de 5 micras, coradas pela técnica rotineira de hematoxilina-eosina e analisadas em microscópio de luz quanto aos seguintes parâmetros histopatológicos: tumefação/congestão, necrose, esteatose microvesicular, proliferação de ductos biliares, infiltrado inflamatório e fibrose.

A pata anterior direita de cada animal abatido foi coletada e colocada em recipiente de alumínio contendo água e fervida por 25 minutos para amolecimento da pele e da carne e retirada do terceiro osso metacarpiano. Os metacarpos foram mantidos em freezer $\left(-12^{\circ} \mathrm{C}\right)$ e, depois, colocados em estufa ventilada a $65{ }^{\circ} \mathrm{C}$ por um período de 72 horas, submetidos à prensagem mecânica e desengordurados em extrator Soxhlet por 4 horas. Posteriormente, os ossos foram levados à estufa $65^{\circ} \mathrm{C}$ por 24 horas. Em seguida, triturados em moinho de bola e acondicionados em vidros identificados, para posteriores análises.

As análises dos teores ósseos de cálcio e fósforo foram realizadas no Laboratório de Nutrição Animal do Departamento de Zootecnia da Universidade Federal de Viçosa (UFV), de acordo com o método descrito por Silva \& Queiroz (2002). 
Os dados das características de desempenho (ganho de peso, consumo de ração e conversão alimentar) e de carcaça (profundidade de lombo, espessura de toucinho, porcentagem e quantidade de carne magra, peso e rendimento de carcaça) e dos parâmetros sanguíneos e ósseos foram submetidos à análise de variância. Os dados dos parâmetros histopatológicos foram submetidos à análise de dados nãoparamétricos de Kruss-Kall Walys. As análises estatísticas foram realizadas utilizando-se o SAEG (Sistemas de Análises Estatísticas e Genéticas, versão 8.0).

\section{Resultados e Discussão}

Durante o período experimental, as temperaturas mínima e máxima médias do ar registradas no galpão foram, respectivamente, $21,5 \pm 2,2{ }^{\circ} \mathrm{C}$ e $29,8 \pm 2,1^{\circ} \mathrm{C}$. Considerando que a temperatura crítica máxima para esta categoria é de $27^{\circ} \mathrm{C}$, conforme descrito por Sampaio et al. (2004), pode-se inferir com base nos desvios térmicos que os animais foram submetidos a períodos de altas temperaturas ambientais durante a execução da pesquisa.

Não houve efeito $(\mathrm{P}>0,05)$ dos níveis de zeólita das dietas sobre o ganho de peso diário dos animais (Tabela 2). Resultados similares foram obtidos por Shurson et al. (1984), que, testando a inclusão de zeólita em dietas para suínos, não verificaram efeitos de níveis de 0,3 e $0,5 \%$ na fase de crescimento e 1,0 e 5,0\% na fase de terminação sobre o desempenho dos suínos em relação ao grupo alimentado com a dieta sem zeólita. Da mesma forma, Ward et al. (1991) não constataram efeito da inclusão de $0,5 \%$ de zeólita em dietas para suínos nas fases iniciais e de crescimento.

Por outro lado, Vrzgula \& Bartko (1984) e Leung (2004) constataram efeito positivo da inclusão de, respectivamente, 3,0 e 4,0\% de zeólita na dieta sobre o ganho de peso de suínos em fase de crescimento e terminação em comparação ao grupo controle. De forma similar, Defang \& Nikishov (2009), avaliando níveis de inclusão de zeólita na dieta de suínos em fase de crescimento e terminação, obtiveram os melhores resultados de crescimento dos animais com a inclusão de 4,0\% de zeólita a dieta.
Os níveis de zeólita não influenciaram $(\mathrm{P}>0,05)$ o consumo diário de ração dos suínos (Tabela 2), resultado que está coerente com os obtidos por Shurson et al. (1984), Leung (2004) e Defang \& Nikishov (2009), que também não observaram variações na ingestão voluntária de alimentos pelos animais atribuível à inclusão de zeólita na dieta.

Não foi observado efeito $(\mathrm{P}>0,05)$ dos níveis de zeólita sobre a conversão alimentar dos animais (Tabela 2). Esses resultados estão consistentes com os obtidos por Shurson et al. (1984) e Defang \& Nikishov (2009), que também não verificaram efeito da inclusão de zeólita sobre a conversão alimentar dos animais. Por outro lado, Vrzgula \& Bartko (1984) e Leung (2004) constataram variação significativa na eficiência de utilização de alimento para ganho de peso dos suínos em crescimento e terminação em razão da inclusão de zeólita na dieta.

Considerando que os animais do tratamento sem adsorvente apresentaram bom índice de desempenho de acordo com preconizado por Rostagno et al. (2005) para essas categorias animal e que, segundo Dersjant-Li et al. (2003), as micotoxinas podem modificar o metabolismo das proteínas, carboidratos e lipídios, o que resulta em redução do despenho dos animais (Agag, 2004), pode-se deduzir que as dietas continham baixo grau de contaminação por micotoxinas. Essa hipótese é confirmada pelos estudos de Dersjant-Li et al. (2003) e Chen et al. (2008), que relataram a necessidade de uma concentração mínima de micotoxina na dieta para que ocorra redução do crescimento dos suínos.

Os níveis de zeólita nas dietas não influenciaram $(\mathrm{P}>0,05)$ as características quantitativas de carcaça avaliadas (Tabela 3). Os resultados estão coerentes com os obtidos por Ward et al. (1991), que, testando inclusão de 0,5\% de zeólita na dieta de suínos em fases de crescimento e terminação, não verificaram efeito do adsorvente sobre o peso de carcaça, a área de olho-de-lombo, o rendimento de carcaça, a espessura de toucinho e a porcentagem de carne magra. Da mesma forma, Pearson et al. (1985), avaliando níveis de 4,0 e 8,0\%, e Leung (2004), testando níveis de 2,0 a 6,0\%, não observaram efeito da inclusão de zeólita na dieta de suínos em fase de crescimento e terminação sobre as

Tabela 2 - Desempenho de suínos em fase de crescimento e terminação alimentados com dietas contendo diferentes níveis de zeólita

\begin{tabular}{lcccccccc}
\hline Parâmetro & \multicolumn{9}{c}{ Nível, \%* } & \multicolumn{3}{c}{ CV, \% } \\
\cline { 2 - 6 } & $0,0-0,0$ & $1,0-0,5$ & $1,0-1,0$ & $2,0-1,0$ & $2,0-2,0$ & $3,0-1,5$ & $3,0-3,0$ \\
\hline Peso inicial, kg & 30,40 & 29,99 & 30,30 & 30,39 & 30,40 & 30,17 & 30,73 & 3,59 \\
Peso final, kg & 98,27 & 96,95 & 98,60 & 99,87 & 97,02 & 97,85 & 97,66 & 3,36 \\
Consumo de ração diário, g & 2.526 & 2.520 & 2.467 & 2.546 & 2.580 & 2.476 & 2.466 & 5,95 \\
Ganho de peso diário, g & 1.044 & 1.029 & 1.050 & 1.068 & 1.024 & 1.040 & 1.028 & 4,55 \\
Conversão alimentar & 2,41 & 2,44 & 2,34 & 2,38 & 2,51 & 2,38 & 2,39 & 5,78 \\
\hline
\end{tabular}

* Níveis de zeólita, respectivamente, nas fases de crescimento e terminação. 
características de carcaça quando comparados ao tratamento controle.

Em contrapartida, Defang \& Nikishov (2009) constataram maior rendimento de carcaça para os suínos alimentados com dietas contendo 4,0\% de zeólita, porém sem alterações as demais características de carcaça. Por sua vez, Pond et al. (1988) constataram aumento da área de olho-de-lombo com a inclusão de zeólita na dieta.

Conforme relato de Szkudelska et al. (2002), a zearalenona acima de uma concentração mínima de $1 \mathrm{mg} / \mathrm{kg}$ interfere com metabolismo dos lipídios, reduzindo a lipólise. Como neste estudo não foi observada variação significativa na espessura de toucinho dos animais entre os tratamentos, confirma-se a proposição inicial do baixo nível de contaminação por micotoxinas das dietas, entre elas, a zearalenona.

Os resultados observados para as características de carcaça deste estudo estão coerentes com as respostas de desempenho, em que não houve efeitos sobre a taxa de crescimento e conversão alimentar dos animais em função dos níveis de zeólita avaliados.

Não houve efeito $(\mathrm{P}>0,05)$ dos níveis de zeólita na dieta sobre os parâmetros sanguíneos dos suínos (Tabela 4), que se mantiveram dentro da faixa de normalidade, independentemente dos tratamentos. Estes resultados estão semelhantes aos obtidos por Watts et al. (2003), que não constataram alteração significativa nos parâmetros sanguíneos de aves com a inclusão de 1,0\% de zeólita na dieta em comparação às aves que receberam a dieta sem adsorvente.

De forma contrária, Harvey et al. (1989) observaram que a inclusão de 0,5 e 2,0\% de adsorvente à dieta de suínos em fase de crescimento foi efetiva para manter normais os parâmetros sanguíneos estudados. De acordo com esses autores, o resultado comprovou que a adição de adsorvente à dieta foi efetiva para limitar a absorção de micotoxinas pelo trato gastrintestinal. Posteriormente, Lindemann et al. (1993), trabalhando com leitões nas fases iniciais de crescimento, constataram redução dos níveis sanguíneos de bilirrubina e aspartato aminotransferase e aumento da glicose, albumina e ureia circulante quando incluíram 0,5\% de zeólita sintética a dietas contaminadas. Os autores concluíram que a alteração nos parâmetros sanguíneos observados seria resultante dos efeitos adversos das micotoxinas sobre o metabolismo proteico.

A divergência de resultados entre os trabalhos pode estar relacionada, entre outros fatores, à diferença no nível de contaminação das dietas. Em trabalho conduzido com leitões, Accensi et al. (2006) verificaram que baixos níveis de micotoxinas na dieta não são suficientes para causar efeitos negativos sobre o desempenho e os parâmetros sanguíneos e histológicos do parênquima hepático dos animais.

Tabela 3 - Características de carcaça de suínos em fase de crescimento e terminação alimentados com dietas contendo diferentes níveis de zeólita

\begin{tabular}{|c|c|c|c|c|c|c|c|c|}
\hline \multirow[t]{2}{*}{ Parâmetro } & \multicolumn{7}{|c|}{ Nível, \%* } & \multirow[t]{2}{*}{$\mathrm{CV}, \%$} \\
\hline & $0,0-0,0$ & $1,0-0,5$ & $1,0-1,0$ & $2,0-1,0$ & $2,0-2,0$ & $3,0-1,5$ & $3,0-3,0$ & \\
\hline Espessura de toucinho, mm & 14,7 & 15,9 & 14,3 & 14,6 & 13,6 & 13,8 & 13,7 & 16,61 \\
\hline Profundidade de lombo, mm & 53,0 & 52,0 & 57,5 & 57,7 & 55,7 & 58,1 & 56,2 & 9,83 \\
\hline Carne magra, \% & 55,8 & 54,4 & 55,3 & 55,9 & 56,7 & 56,5 & 56,4 & 3,34 \\
\hline Carne magra, kg & 41,0 & 39,7 & 40,0 & 43,8 & 41,7 & 41,8 & 41,6 & 5,93 \\
\hline Peso de carcaça, kg & 76,0 & 74,5 & 73,5 & 75,0 & 75,7 & 74,8 & 74,8 & 4,36 \\
\hline Rendimento carcaça, \% & 74,0 & 72,0 & 73,0 & 74,4 & 72,9 & 73,5 & 74,4 & 2,84 \\
\hline
\end{tabular}

* Níveis de zeólita, respectivamente, nas fases de crescimento e terminação.

Tabela 4 - Parâmetros sanguíneos de suínos em fase de crescimento e terminação alimentados com dietas contendo diferentes níveis de zeólita

\begin{tabular}{|c|c|c|c|c|c|c|c|c|}
\hline \multirow[t]{2}{*}{ Parâmetro } & \multicolumn{7}{|c|}{ Nível, \%* } & \multirow[t]{2}{*}{$\mathrm{CV}, \%$} \\
\hline & $0,0-0,0$ & $1,0-0,5$ & $1,0-1,0$ & $2,0-1,0$ & $2,0-2,0$ & $3,0-1,5$ & $3,0-3,0$ & \\
\hline Alanina aminotransferase & 37,75 & 37,00 & 37,91 & 38,91 & 37,58 & 40,81 & 38,30 & 15,94 \\
\hline Aspartato aminotransferase & 32,81 & 31,81 & 28,5 & 30,66 & 29,66 & 32,36 & 28,58 & 18,41 \\
\hline Albumina & 4,14 & 4,24 & 4,14 & 4,18 & 4,21 & 4,17 & 4,17 & 4,45 \\
\hline Proteína total & 74,50 & 75,42 & 73,40 & 71,89 & 74,60 & 73,88 & 72,64 & 5,54 \\
\hline Ureia & 34,02 & 32,80 & 30,93 & 36,05 & 32,40 & 33,66 & 35,55 & 22,55 \\
\hline Creatinina & 1,35 & 1,36 & 1,33 & 1,35 & 1,3 & 1,37 & 1,39 & 9,76 \\
\hline Fosfatase alcalina & 206,18 & 190,27 & 177,83 & 190,09 & 180,09 & 199,54 & 186,30 & 23,58 \\
\hline Gama glutamil transferase & 48,45 & 41,72 & 45,58 & 38,08 & 38,41 & 45,40 & 41,18 & 22,81 \\
\hline Glicose & 109,16 & 108,62 & 106,87 & 107,31 & 106,05 & 109,64 & 104,38 & 10,10 \\
\hline
\end{tabular}

* Níveis de zeólita, respectivamente, nas fases de crescimento e terminação. 
Os níveis de zeólita não influenciaram os parâmetros ( $\mathrm{P}>0,05)$ histológicos do parênquima hepático avaliado (Tabela 5). Contudo, Harvey et al. (1994), trabalhando com suínos nas fases iniciais de crescimento, constataram redução das lesões hepáticas dos animais que receberam dietas contaminadas com micotoxinas contendo $0,5 \%$ de zeólita em comparação aos animais que receberam dietas contaminadas e não suplementadas. Do mesmo modo, Denli et al. (2009) notaram redução das lesões hepáticas em frangos de corte com a inclusão de adsorvente na dieta.

O fato de neste estudo não ter sido observada influência de zeólita sobre os parâmetros histológicos do parênquima hepático pode ser justificado pela normalidade dos valores desses parâmetros nos animais que receberam dieta sem zeólita.

Os conteúdos de cálcio e fósforo nos ossos dos animais não foram influenciados $(\mathrm{P}>0,05)$ pelos níveis de zeólita da dieta (Tabela 6). Esses resultados são semelhantes aos parâmetros ósseos obtidos por Ward et al. (1991) e corroboram os obtidos por Lindmann et al. (1993), Harvey et al. (1994) e Watts et al. (2003), que não constataram efeito da zeólita sobre as concentrações séricas de cálcio e fósforo. A partir desses resultados, é possível inferir que a zeólita não influencia a digestibilidade e absorção de cálcio e fósforo das dietas.

Tabela 5 - Escores de lesões ( 0 = ausência, 1 = leve, 2 = moderada e 3 = severa) histopalógicas do parênquima hepático de suínos em fase de crescimento e terminação alimentados com diferentes níveis de zeólita

\begin{tabular}{|c|c|c|c|c|c|c|c|}
\hline \multirow[t]{2}{*}{ Parâmetro } & \multicolumn{7}{|c|}{ Nível, \%* } \\
\hline & $0,0-0,0$ & $1,0-0,5$ & $1,0-1,0$ & $2,0-1,0$ & $2,0-2,0$ & $3,0-1,5$ & $3,0-3,0$ \\
\hline Tumefação/congestão & 1,15 & 0,90 & 0,58 & 0,67 & 0,75 & 0,45 & 0,92 \\
\hline Necrose & 0,25 & 0,91 & 0,83 & 0,00 & 0,00 & 0,00 & 0,00 \\
\hline Esteatose microvesicular & 0,25 & 0,18 & 0,25 & 0,25 & 0,33 & 0,18 & 0,00 \\
\hline Proliferação de ductos biliares & 0,83 & 1,10 & 1,08 & 0,67 & 0,83 & 0,91 & 0,50 \\
\hline Infiltrado inflamatório & 0,42 & 0,64 & 0,67 & 0,34 & 0,83 & 0,64 & 0,42 \\
\hline Fibrose & 0,92 & 1,09 & 0,67 & 0,50 & 0,58 & 0,91 & 0,58 \\
\hline
\end{tabular}

*Níveis de zeólita, respectivamente, nas fases de crescimento e terminação.

Tabela 6 - Concentrações de cálcio e fósforo nos ossos de suínos alimentados com dietas com diferentes níveis de zeólita

\begin{tabular}{lcccccccc}
\hline Parâmetro & \multicolumn{9}{c}{ Nível, \%* } & \multicolumn{3}{c}{ CV, \% } \\
\cline { 2 - 7 } & $0,0-0,0$ & $1,0-0,5$ & $1,0-1,0$ & $2,0-1,0$ & $2,0-2,0$ & $3,0-1,5$ & $3,0-3,0$ \\
\hline Cálcio, g/kg & 181,35 & 177,61 & 174,27 & 182,17 & 177,3 & 183,02 & 174,65 & 9,93 \\
Fósforo, g/kg & 93,85 & 95,84 & 90,79 & 93,24 & 92,37 & 94,51 & 93,46 & 8,85 \\
\hline
\end{tabular}

* Níveis de zeólita, respectivamente, nas fases de crescimento e terminação.

\section{Conclusões}

Os níveis de zeólita não influenciam o desempenho e as características de carcaça nem os parâmetros sanguíneos e histológicos do parênquima hepático de suínos em fase de crescimento e terminação quando alimentados com dietas de baixo nível de contaminação por micotoxinas.

\section{Referências}

ACCENSI, F.; PINTON, P.; CALLU, P. et al. Ingestion of low doses of deoxynivalenol does not affect hematological, biochemical, or immune responses of piglets. Journal of Animal Science, v.84, p.1935-1942, 2006.

AGAG, B.I. Mycotoxins in foods and feeds. 1-aflatoxins. Association University Bull Environment Research, v.7, n.1, p.173-206, 2004
BÜZEN, S.; HAEZE, D. Controle de micotoxinas na alimentação de aves e suínos. Revista Eletrônica Nutritime, v.3, n.1, p.299-304, 2006.

CHEN, F.; MA, Y.L.; XUE, C.Y. et al. The combination of deoxynivalenol and zearalenone at permitted feed concentrations causes serious physiological effects in young pigs. Journal of Veterinary Science, v.9, p.39-44, 2008.

DEFANG, H.F.; NIKISHOV, A.A. [2009]. Effect of dietary inclusion of zeolite on performance and carcass quality of grower-finisher pigs. Livestock Research for Rural Development, v.21, n.6, 2009. Disponível em: <http://www.lrrd.org/lrrd21/6/defa21090.htm> Acesso em: 21 jan. 2010.

DENLI, M.; BLANDON, J.C.; GUYNOT, M.E. et al. Effects of dietary AflaDetox on performance, serum biochemistry, histopathological changes and aflatoxin residues in broilers exposed to aflatoxin $\mathrm{B}_{1}$. Poultry Science, v.88, p.1444-1451, 2009.

DERSJANT-LI, Y.; VERSTEGEN, M.W.A.; GERRITS, W.J.J. The impact of low concentrations of aflatoxin, deoxynivalenol or fumonisins in diets on growing pigs and poultry. Nutrition Research Reviews, v.16, p.223-239, 2003.

HARVEY R.B.; KUBENA L.F.; PHILLIPS T.D. et al. Prevention of aflatoxicosis by addition of hydrated sodium calcium 
aluminosilicate to the diets of growing barrows. American Journal of Veterinary Research, v.50, p.416-420, 1989.

HARVEY, R.B.; KUBENA, L.F.; ELISSALDE, M.H. et al. Comparison of two hydrated sodium calcium aluminosilicate compounds to experimentally protect growing barrows from aflatoxicosis. The Journal of Veterinary Diagnostic Investigation, v.6, p.88-92, 1994.

JONES, F.T. Is mold growth hurting your performance? Avian Advice, v.7, n.1, p.8-11, 2005. Disponível em: <http://www.avianadvice.uark.edu/ AA\%20PDFs/Avian_AdviceWi2005.pdf> Acesso em: 12 jul. 2009.

LEUNG, S. The effect of clinoptilolite properties and supplementation levels on swine performance. 2004. 131f. Thesis (Master in Bioresource Engineering) - McGill University, Montreal.

LINDEMANN, M.D.; BLODGETT, D.J.; KORNEGAY, E.T. Potential ameliorators of aflatoxicosis in weanling-growing swine. Journal of Animal Science, v.71, p.171-178, 1993.

MURPHY, P.A.; HENDRICH, S.; LANDGREN, C. et al. Food mycotoxins: an update. Journal of Food Science, v.71, n.5, p.51-65, 2006.

PEARSON, G.; SMITH, W.C.; FOX, J.M. Influence of dietary zeolite on pig performance over the liveweight range 25-87 kg. New Zealand Journal of Experimental Agriculture, v.13, p.151-154, 1985.

POND, W.G.; YEN, J.T.; VAREL, V.H. Response of growing swine to dietary copper and clinoptilolite supplementation. Nutrition Reports International, v.37, p.795-803, 1988.

ROSTAGNO, H.S.; ALBINO, L.F.T.; DONZELE, J.L. et al. Tabelas brasileiras para aves e suínos: composição de alimentos e exigências nutricionais. 2.ed. Viçosa, MG: Universidade Federal de Viçosa, 2005. 186p.
RUSTEMEYER, S.M.; LAMBERSON, W.R.; LEDOUX, D.R. et al. Effects of dietary aflatoxin on the health and performance of growing barrows. Journal of Animal Science, v.88, p.3624-3630, 2010.

SAMPAIO, C.A.P.; CRISTANI, J.; DUBIELA, J.A. et al. Avaliação do ambiente térmico em instalações para crescimento e terminação de suínos utilizando os índices de conforto térmico nas condições tropicais. Ciência Rural, v.34, p.785-790, 2004.

SHURSON, G.C.; KU, P.K.; MILLER, E.R. et al. Effects of zeolite A or clinoptilolite in diets of growing swine. Journal of Animal Science, v.59, p.1536-1545, 1984.

SILVA, D.J.; QUEIROZ, A.C. Análise de alimentos: métodos químicos e biológicos. 3.ed. Viçosa, MG: Universidade Federal de Viçosa, 2002. 235p.

SZKUDELSKA, K.; SZKUDELSKI, T.; NOGOWSKI, L. Daidzein, coumestrol and zearalenone affect lipogenesis and lipolysis in rat adipocytes. Phytomedicine, v.9, n.4, p.338-345, 2002.

VRZGULA, L.; BARTKO, P. Effect of clinoptilolite on weight gain and some physiological parameters of swine. In: POND, W.G.; MUMPTON, F.A. (Eds.) Zeo-agriculture. Brockport, New York: International Community of Natural Zeolites, 1984. p.161-166.

WARD, T.L.; WATKINS, K.L.; SOUTHERN, L.L. et al. Interactive effects of sodium zeolite-A and copper in growing swine: growth, and bone and tissue mineral concentrations. Journal of Animal Science, v.69, p.716-733, 1991.

WATTS, C.M.; CHEN, Y.C.; LEDOUX, D.R. et al. Effects of multiple mycotoxins and a hydrated sodium calcium aluminosilicate in poultry. International Journal of Poultry Science, v.2, n.6, p.372-378, 2003. 